\title{
Les pithoi de Margaritès (Crète)
}

Données techniques et aperçu historique

Philippe Gouin et Christine Vogt

\section{CpenEdition}

Journals

Édition électronique

URL : https://journals.openedition.org/tc/1084

DOI : $10.4000 /$ tc. 1084

ISSN : 1952-420X

Éditeur

Éditions de l'EHESS

\section{Édition imprimée}

Date de publication : 1 mars 2002

ISSN : 0248-6016

\section{Référence électronique}

Philippe Gouin et Christine Vogt, « Les pithoi de Margaritès (Crète) », Techniques \& Culture [En ligne], 38 | 2002, mis en ligne le 11 juillet 2006, consulté le 29 septembre 2022. URL : http://

journals.openedition.org/tc/1084; DOI : https://doi.org/10.4000/tc.1084

Ce document a été généré automatiquement le 29 septembre 2022.

Tous droits réservés 


\title{
Les pithoi de Margaritès (Crète)
}

\author{
Données techniques et aperçu historique
}

Philippe Gouin et Christine Vogt

Le cadre

« Une fois donc, j’ai été potier. Ce métier-là je

l'aimais comme un fou. Tu sais ce que c'est que de prendre une motte de boue et d'en faire tout ce

que tu veux ?... C'est ça qui s'appelle être un homme : Liberté !» (N. Kazantzaki, Alexis Zorba).

1 Implanté sur l'un des éperons rocheux parallèles qui forment les premiers contreforts du mont Psiloritis, en Crète centrale (fig. 1), le site de potiers de Margaritès comporte trois secteurs bien distincts : le village, les ateliers et les carrières. Le village lui-même est bâti sur l'extrême pointe de l'éperon et bordé de profonds ravins. Il abrite des habitations et des ateliers-boutiques pour touristes. Les ateliers de potiers traditionnels, partiellement abandonnés aujourd'hui, sont implantés à une certaine distance du village, là où l'éperon s'élargit et forme un large plateau rocheux. Les carrières d'argile, enfin, sont situées encore plus au sud, sur les pentes mêmes du Psiloritis. Chargés d'étudier la céramique protobyzantine de la cité antique d'Eleutherna, à moins de deux kilomètres de là (Thémélis 2000), nous ne pouvions donc ignorer l'existence de cet important complexe potier ${ }^{1}$.

2 Sorte de Vallauris crétois, Margaritès est aujourd'hui relié par des routes modernes à Réthymno et à Héraklion, villes portuaires et balnéaires de la côte nord. À la belle saison, il y a donc forte affluence de touristes dans le village où des " céramistes d'art " ont remplacé les vieux potiers d'antan (Gouin \& Vogt 1996). La visite du site n'aurait qu'un intérêt limité si deux maîtres artisans, M. Nikos Kavgalakis et M. Manolis, n'avaient continué à produire de la poterie traditionnelle dans leurs ateliers isolés. De ces deux artisans, seul le premier produit encore les grands vases de stockage, les pithoi (se prononce pithi en grec moderne), selon des techniques immémoriales, le second ne tournant plus que de petites pièces culinaires, cruches, pots, amphorettes ou gourdes. Dès notre première visite, nous avons donc compris que s'offrait à nous une exceptionnelle occasion d'étudier la fabrication de ces grands vases aux origines très 
anciennes dont la technique de façonnage a souvent fait l'objet de controverses chez les archéologues ${ }^{2}$.

De l'écuelle au presse-citron et de la tirelire au baptistère, presque tous les ustensiles domestiques des familles et des villages de la région étaient autrefois façonnés à Margaritès (Vallianos \& Padouva 1986). Éléments essentiels de cette production si variée, les pithoi, à la fois tonneaux, coffres et silos, constituaient les supports de l'économie rurale (fig. 2).

4 Ces vases, intransportables lorsqu'ils sont pleins, sont donc "statiques » et attachés aux foyers qui les abritent. Déjà présents dans les habitations antiques, ils deviennent très communs dans les maisons villageoises dès le début de la période médiévale et le restent durant une douzaine de siècles, jusqu'à nos jours. Ce phénomène correspond au brusque changement de société qui suivit l'abandon, au VIIe siècle, des cités antiques de Crète centrale et la fuite d'une partie de leur population devant l'avancée arabe. Les Crétois restants s'installèrent dans des villages inexpugnables construits sur les anciennes acropoles. Il en résulta un profond bouleversement de la société et de l'économie (Vogt 2000). En effet, si sur leurs pitons, les montagnards crétois se trouvèrent à l'abri des pirates et des conquérants, ils se placèrent aussi à l'écart des circuits commerciaux internationaux et même régionaux. Partiellement vidée de ses forces dynamiques, la Crète resta ainsi isolée des grands courants d'échanges durant plusieurs siècles.

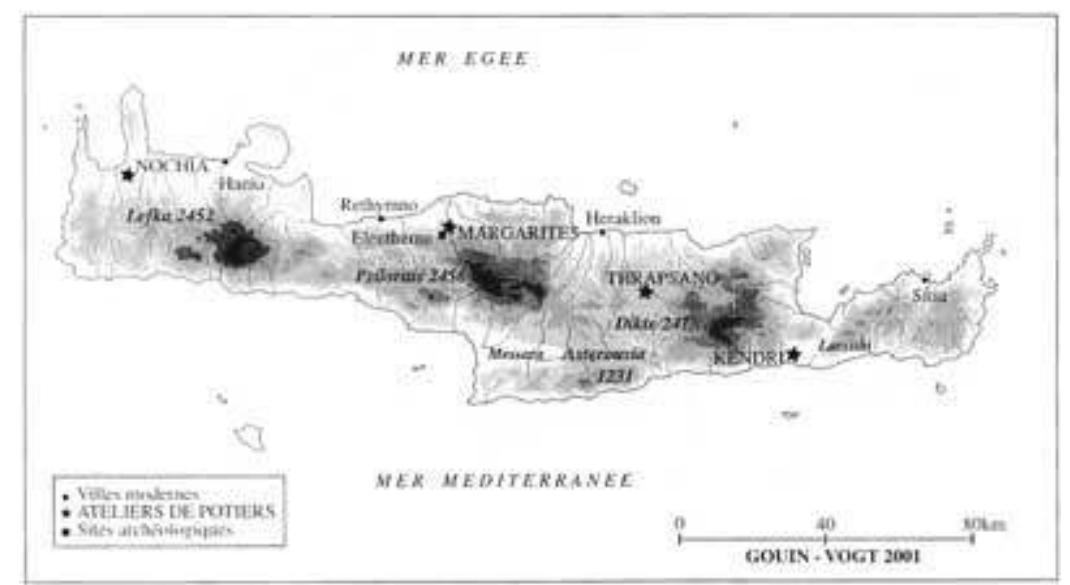


Figure 1. Carte des principaux centres potiers (dessin $\mathrm{Ch}$. Vogt et $\mathrm{Ph}$. Gouin)

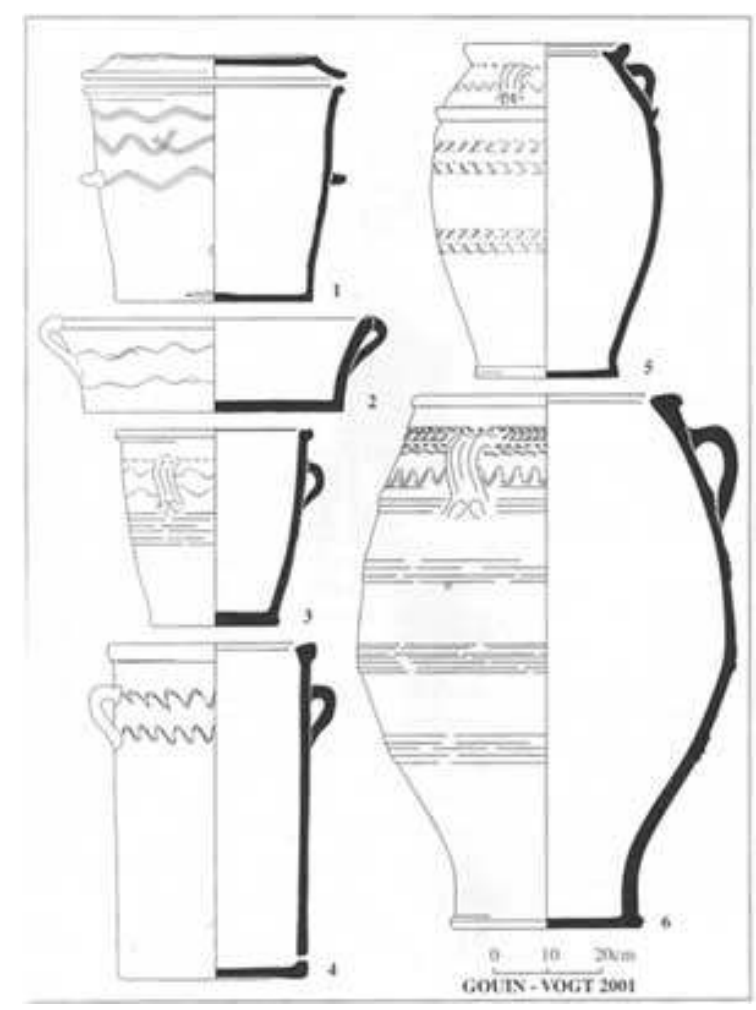

Figure 2. Examples de grands vases traditionnels : 1 . ruche à couvercle; 2 . pétrin; 3 . cuve à lessive; 4. jarre à fromages; 5 . jarre à miel; 6 . grande jarre à 3 anses (dessin de Ch. Vogt et $\mathrm{Ph}$. Gouin, Ethnographic Museum, Réthymno)

5 L'élevage du menu bétail (ovins, caprins) et des abeilles, la culture des oliviers, de la vigne et des légumes, facilement praticables sur les terrains pauvres et accidentés de la montagne, devinrent alors primordiaux dans l'économie rurale. Limitée par l'exiguïté des terres exploitables sur les pitons, la production des villages n'alla guère au-delà de ce qui était nécessaire à leurs besoins (Gouin \& Vogt 2000). Lorsqu'au Xe siècle, l'île fut reprise par les Byzantins, les villages de l'intérieur s'étaient organisés et, ne comptant plus que sur eux-mêmes, vivaient assez chichement en économie autarcique. Les habitudes étant prises, ni la relance du commerce, ni la reprise des exportations ne changèrent le mode de vie adopté par les Crétois durant les siècles sombres. Chaque famille continua à constituer individuellement les réserves de denrées de base: vin, huile, céréales, viande salée, légumes en saumure, etc., qui lui permettaient de subsister durant les saisons improductives. Cette tendance à l'accumulation alimentaire s'accompagna évidemment d'une augmentation des besoins en vases de stockage et, en particulier, en pithoi. Les potiers locaux durent alors développer leur production en conséquence. Tout cela nous fait dire que c'est aux XIIIe et XIVe siècles, sous la prospère occupation vénitienne, que le village de Margaritès prit l'importance régionale qu'il a conservée jusqu'à nos jours. Depuis, toute famille crétoise se doit de posséder au moins deux ou trois de ces monuments dans lesquels elle conserve, à l'abri des agressions du biotope, ses humbles trésors, des olives salées aux vêtements de cérémonie. Même fendus, les pithoi trouvent une seconde vie comme conduits de cheminées, coffrages de puits, drains, niches à chiens... ${ }^{3}$. 
6 Il a pourtant suffi de deux ou trois décennies pour que la poterie domestique disparaisse presque complètement au profit du fer étamé ou émaillé, puis de l'aluminium et enfin de l'acier inoxydable et de la matière plastique. La modernisation des routes et l'installation de l'électricité et de l'eau courante sont sans doute les principaux facteurs de cette éclipse. En effet, la construction d'équipements modernes à partir des années soixante a conduit au désenclavement des villages et au développement des échanges avec les côtes. Ceux-ci ont permis aux villageois les plus aisés d'acquérir des machines domestiques et agricoles modernes dorénavant utilisables. En vingt ans, les villages passent ainsi d'une économie fermée à une économie d'échange qui, comme ailleurs, s'accompagne de l'abandon des traditions et des artisanats. Le départ des jeunes vers les villes côtières ou le continent complète ce classique processus d'évolution. Les goûts et les besoins alimentaires s'en trouvent aussi bouleversés. Bien qu'avec beaucoup de réticences, le paysan crétois est peu à peu amené à copier les usages alimentaires transmis par les centres urbains. Pourquoi continuer à faire soi-même son pain et à le manger rassis alors que le bus l'apporte chaque jour frais du bourg voisin? Pourquoi préparer des olives, de l'huile et des fromages puisque, depuis le départ des jeunes pour les villes, seuls les vieux en mangent encore? Pourquoi alors conserver les énormes jarres moisies qui encombrent la cave de la maison au lieu de les vendre aux « antiquaires » d'Athènes? Ainsi, dans quelques années, plus aucun pithos de Crète n'aura d'usage alimentaire. Cependant, tout en regrettant leur déclin, nous reconnaîtrons que les pithoi présentent d'importants inconvénients fonctionnels. En effet, ces vases lourds et fragiles sont difficiles à manipuler et leur fabrication consomme beaucoup d'argile (de 60 à 80 kilos par pithos) et de combustible. Poreux et grossiers, ils se nettoient difficilement ${ }^{4}$ et sont bien moins attrayants que les tonneaux en matière plastique multicolore ${ }^{5}$. Nul doute donc que l'arrivée de ces rutilants vases industriels ne constitue un réel progrès pour le paysan, même si elle annonce la fin de traditions millénaires ${ }^{6}$.

Les ateliers

7 L'évidence et de nombreux indices archéologiques nous indiquent que les potiers s'installèrent sur le site de Margaritès lorsque la ville antique d'Eleutherna fut fondée, c'est-à-dire au plus tard au milieu du premier millénaire avant notre ère ${ }^{7}$. Les premiers habitants de cette très importante cité ne pouvaient en effet ignorer qu'il existait des carrières d'argile à pots à une demi-heure de marche de leurs murs. L'hypothèse de l'exploitation et de l'occupation antique de ce site est d'ailleurs, à notre avis, confirmée par les analyses comparatives de pâtes réalisées par le Centre Démokritos à Athènes (Vogt 2000; Aloupi et al. 2000). Nous ne doutons donc pas qu'une soigneuse prospection permettrait de retrouver, près des ateliers abandonnés du siècle dernier, des traces d'établissements vieux de vingt-cinq siècles. Ainsi, le maître potier Nikos Kavgalakis pourrait bien représenter le dernier maillon d'une longue chaîne d'artisans dont les premiers fournissaient la cité antique. De plus, même s'ils sont abandonnés depuis deux décennies et que les tours et les fours sont envahis par les figuiers et les ronces, les anciens ateliers sont restés à peu près intacts et leur observation complète parfaitement celle du travail des derniers occupants du site (fig. 3). L'ensemble représente donc un modèle ethno-archéologique d'un rare intérêt ${ }^{8}$. 


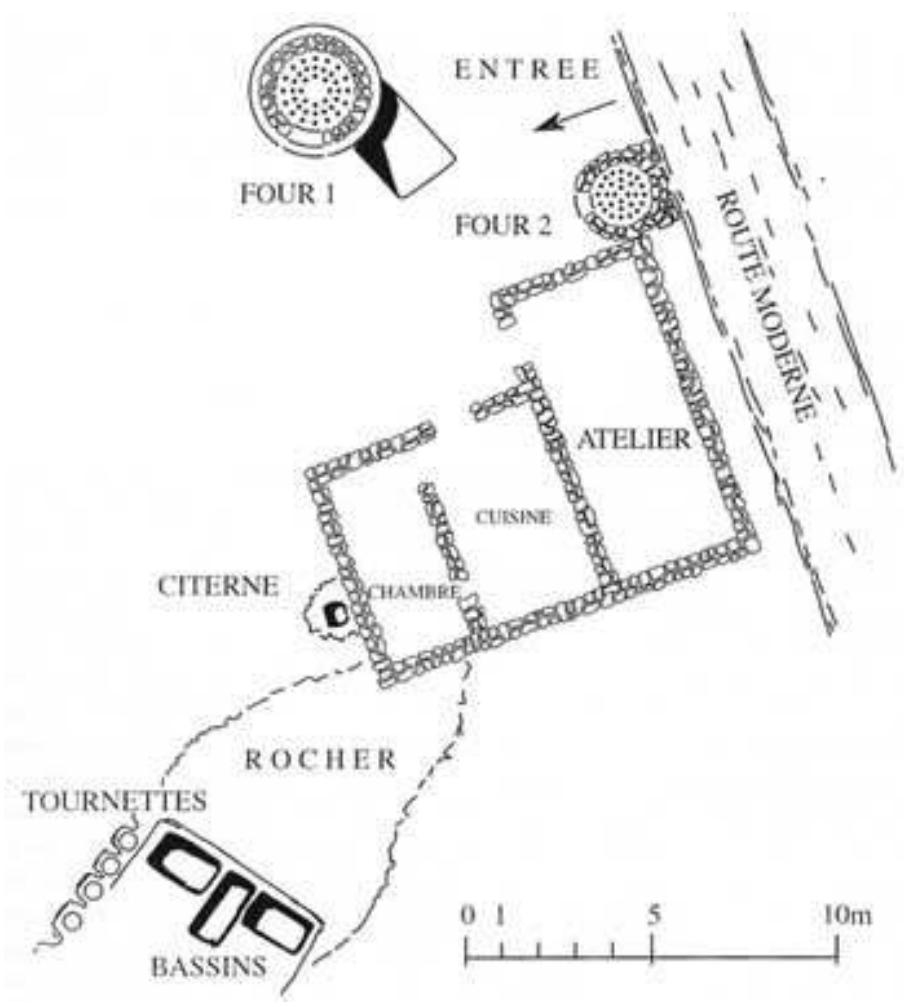

Figure 3. Ancienne installation de potiers (dessin de Ch. Vogt, d'après Vallianos \& Padouva 1986 : 105)

8 Avant de passer à la description des matériels et des techniques, notons qu'autrefois, nombre de potiers étaient aussi paysans et n'exerçaient leur art que durant les mois chauds, d'avril à octobre. En outre, certains d'entre eux étaient itinérants et se déplaçaient de village en village en fonction des demandes de la clientèle. Précisons aussi que Margaritès n'est que l'un des nombreux centres potiers de Crète. Il s'en trouvait jadis dans chaque grande région de l'île. Ceux de Thrapsano au sud d'Héraklion, de Nochia à l'ouest de Hania et de Kendri près d'Iérapétra sont d'ailleurs encore partiellement en activité (voir fig. 1). De même, il en existe plusieurs autres dans l'archipel égéen et sur le continent, mais rares sont ceux qui produisent encore des grands vases traditionnels, cuveaux, bassins et surtout, des jarres (Hampe et Winter 1962; Jones 1986; Blitzer 1990...).

La fabricationPréparation de la pâteExtraction, concassage, criblage des terres

On peut se demander si ce n'est pas uniquement parce qu'il s'y trouvait des carrières d'argile et de pierre, que les Minoens puis les Grecs choisirent le site d'Eleutherna pour $y$ construire une ville (Gouin \& Vogt 2000). En effet, révélées par l'érosion ou le travail des hommes, les carrières d'argile de Margaritès se présentent sous la forme de petites poches particulièrement accessibles qui affleurent la surface du piémont. Chacune d'entre elles contient tout au plus une dizaine de mètres cubes d'argile enrobée dans des terres et des roches friables inutilisables. Une fois épuisée, une poche est abandonnée pour une nouvelle exploitation'. Du fait de l'exiguïté des filons, l'argile doit souvent être extraite à la pioche. Dure, mais friable, elle se détache en petits blocs qui peuvent être transportés en sacs. Élargis, les chemins autrefois réservés aux ânes ${ }^{10}$ autorisent maintenant le transport de la terre par camion jusqu'aux ateliers où elle est stockée en plein air, sur Lin «terrier ». Elle s'y bonifie ainsi durant plusieurs semaines 
ou plusieurs mois. Comme ailleurs, la terre à pots qu'utilisent les potiers de Margaritès résulte du mélange de plusieurs argiles communes locales dans des proportions variables. La principale composante de la pâte est une argile bleue très fine et très grasse, nommée lépida, qui ne contient pratiquement aucun corps étranger. Très pure et très collante, elle doit être mélangée avec une terre sableuse jaunâtre, locale elle aussi, nommée koumoulé. Le mélange peut aussi être additionné d'une certaine quantité d'une troisième terre sableuse rougeâtre appelée kokkinochoma. De gris bleuté lorsqu'il est cru, le mélange devient rouge clair après cuisson ${ }^{11}$.

10 Avant de pouvoir être utilisée, la terre koumoulé jaune, qui forme le dégraissant de la pâte, doit être triée et réduite en granulés. Ce travail était autrefois réalisé à grand peine par le potier. Après avoir étalé une mince couche de terre sur le sol rocheux, celui-ci en retirait les plus gros éléments étrangers, cailloux, racines et débris divers. Il frappait ensuite la terre à toute volée avec la partie plane d'un battoir de bois fait d'une demi-bûche fixée sur un manche courbe (Hampe \& Winter 1962: pl. 1.1; Vallianos \& Padouva 1986: 77, fig. 8). Grossièrement concassée, l'argile était ensuite tamisée à travers un crible dont les mailles -d'environ un centimètre- retenaient les grosses mottes. Celles-ci étaient écrasées jusqu'à ce que toute la terre puisse traverser le tamis. Aujourd'hui, ces opérations sont réalisées en peu de temps par un concasseur-cribleur électrique qui transforme directement la terre brute en un fin granulé prêt pour la lévigation.

Détrempage, lévigation, pourrissage

11 Le détrempage et le décantage, ou " lévigation », permettent d'éliminer les dernières impuretés de l'argile et d'obtenir une matière première homogène. Cette opération est réalisée dans de grands bassins remplis d'eau. Le détrempage sert d'abord à séparer les argiles des quelques éléments lourds qui restent après criblage. Les bassins sont au nombre de deux ou trois. Aujourd'hui en maçonnerie, ils étaient autrefois creusés directement dans le sol rocheux des ateliers. Le premier bassin reçoit les terres broyées. Après un fort brassage, les argiles y déposent leurs éléments lourds, cailloux et graviers. La couche supérieure des boues liquides passe ensuite dans des tamis qui, jadis, étaient faits de peaux de moutons percées de trous. Puis l'argile est conduite dans un deuxième bassin où elle se dépose lentement, et parfois dans un troisième. Lorsque l'eau est devenue claire, le bassin est vidé et l'épaisse couche d'argile décantée sèche partiellement avant d'être découpée en pavés puis stockée. Le moment venu, ces blocs durcis seront de nouveau broyés pour être mélangés aux autres éléments de la pâte dans des proportions variables. La couche d'argile très sableuse qui reste dans le fond des bassins après lévigation était autrefois employée pour la fabrication d'éléments de construction : tuiles, tuyaux, briques, etc.

12 Très pure, la lépida bleue n'a pas besoin d'être décantée. Concassée et finement tamisée, elle est ajoutée telle quelle à la koumoulé jaune, dans la proportion moyenne de $60 \%$ de lépida tamisée pour $40 \%$ de koumoulé

décantée. Le mélange d'argiles, autrefois homogénéisé par un long piétinement de la masse par le potier, le "marchage» (Hampe \& Winter 1962: pl. 1.5), est obtenu aujourd'hui avec des mélangeurs électriques. Recouverte d'une bâche pour éviter le dessèchement, la pâte est ensuite stockée plus ou moins longtemps dans un lieu humide et frais où elle acquiert une viscosité uniforme et se bonifie : c'est le "pourrissage ». Au moment voulu, on lui rend facilement sa plasticité par battage et pétrissage. 

s'améliorèrent considérablement lorsque l'eau courante fut installée dans le village vers 1960. L'eau provenait jusque-là des sources voisines et surtout de citernes, d'une capacité ce 20 à 30000 litres, creusées dans le roc sous chaque maison ou atelier. Ces citernes, dont beaucoup servent encore, se remplissent grâce à la récupération des eaux pluviales des toits et du sol. Certaines de ces installations, qui ressemblent en tous points à celles découvertes dans les habitations et les carrières hellénistiques d'Eleutherna (Kalpaxis et al. 1994: 28-37), sont sans doute très anciennes et peut-être même antiques (Vallianos \& Padouva 1986 : 5, fig. 7; Gouin \&Vogt 1996: 60). Pourtant, durant les étés très secs, elles s'avéraient parfois insuffisantes et les potiers devaient alors s'approvisionner à grand peine aux sources pérennes jaillissant dans les ravins voisins. Exécuté avec des outres portées par des ânes, le remplissage des bassins de décantation, par exemple, demandait une journée entière.

Façonnage des vasesTour et tournettes

Seuls les petits et moyens récipients (cruches, écuelles, pots, etc.) peuvent être façonnés sur le léger tour à pied à volant de bois des potiers crétois. Les grandes jarres, qui mettent en œuvre plusieurs dizaines de kilos de pâte et peuvent atteindre $1,50 \mathrm{~m}$ de haut, sont ébauchées par parties au colombin puis mises en forme sur des batteries de tournettes spéciales (photo 1). Ces tournettes à petit plateau sont mues et lancées à la main par un aide grâce à une barre d'acier horizontale qui, traversant l'axe de rotation, sert de manette (fig. 4). C'est en fait le lourd pithos en cours de mise en forme qui fait office de volant d'inertie. La technique de façonnage mixte des très grands vases au colombin, puis à la tournette, est aussi ancienne yu1 universelle. Les potiers la pratiquent non seulement en Crète et ailleurs en Grèce (Hampe \& Winter 1962; Hampe 1963; Voyatzoglou 1974; Vallianos \& Padouva 1986; Blitzer 1990; Psaropoulou 1996...), mais aussi dans tout le Vieux Monde, de l'Espagne à l'Inde (Centlivres-Demont 1971; Rye \& Evans 1976; Brissaud 1982; Roux 1994; Gelbert 1997...), et cela parfois depuis les Âges des Métaux (Gouin 1997).

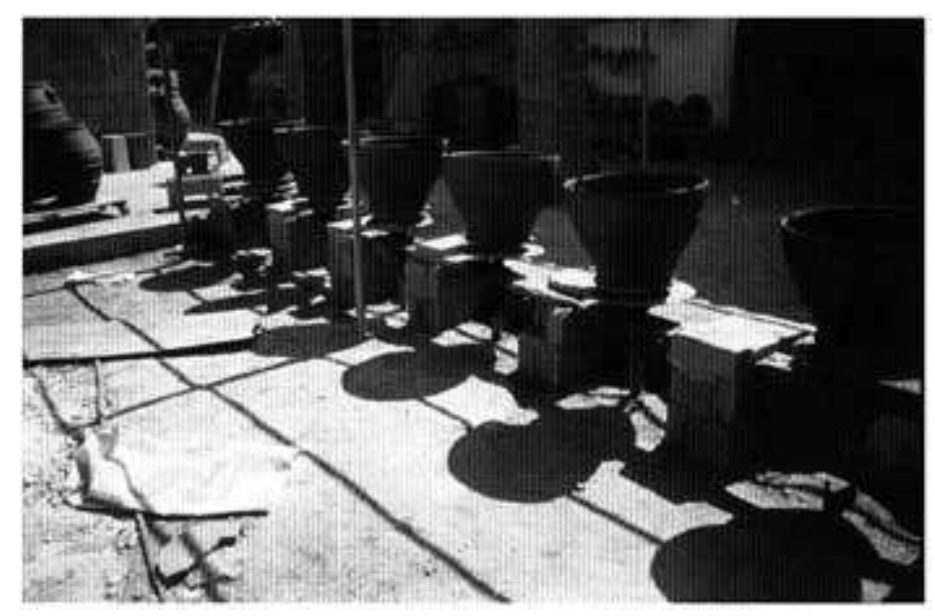

Techniques \& Culture, 38 | 2002 


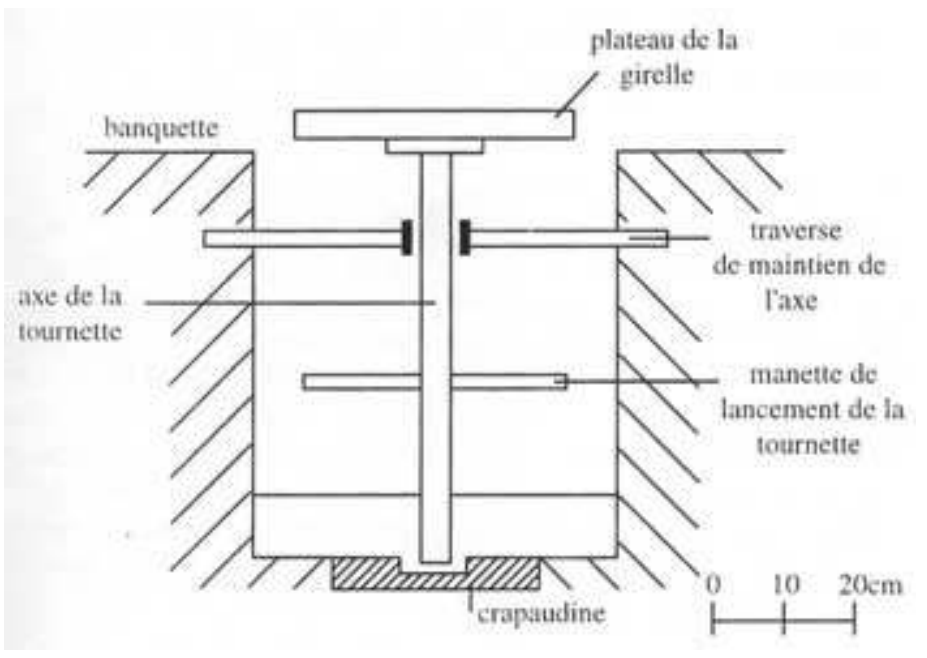

Figure 4. Tournette manuelle (dessin de Ch. Vogt, d'après Vallianos \& Padouva $1986: 105$ )

Façonnage de l'assise et de la base

Pour façonner l'assise de la future jarre, le potier centre sur la girelle le rondeau de schiste ou de céramique individuel qui supportera le vase durant toute sa fabrication et permettra de le déplacer facilement. Il existe donc autant de tournettes et de rondeaux que de vases à monter (photo 2). II pose ensuite une balle de terre humide au centre du disque. Il fait alors tourner lentement la girelle et, du bout des doigts, aplatit la balle pour en faire une galette de deux centimètres d'épaisseur qu'il mesure avec une pointejauge et régularise avec une estèque. Cette galette, lisse et parfaitement circulaire, constitue l'assise sur laquelle le potier monte la base du vase. Pour cela, il prélève une grosse balle d'argile dans le tas qui est près de lui. Il la partage, la malaxe et la frappe entre ses mains pour lui rendre sa plasticité. Il en fait ensuite un gros boudin de 60 à 80 centimètres de long qu'il pose sur le bord de la galette qui tourne lentement sur la girelle (photo 3). Il l'étire et l'amincit par pressions successives du bout des doigts et forme le premier élément de la paroi (photo 4). Durant l'opération, il humidifie la pâte à l'aide d'une éponge et retire les quelques graviers ayant échappé au criblage. Lorsque le premier étage de la base atteint une vingtaine de centimètres de hauteur, il en régularise l'extérieur et le met en forme par un raclage avec l'estèque (photo 5). Encore mou, ce premier élément de la paroi ne pourrait supporter de suite le poids d'un second. Aussi, il est mis à durcir sur son rondeau individuel jusqu'à ce qu'il ait acquis la consistance dite « du cuir ». Pendant ce temps, un autre disque prend sa place sur la girelle du tour pour recevoir une nouvelle base.

Ouvrons ici une parenthèse sur les avantages et les inconvénients du tour électrique. Bien que celui-ci apporte un certain confort au potier pour façonner les premiers étages du vase, il présente pourtant le grave inconvénient d'accroître sensiblement les manutentions. En effet, au lieu de rester en place sur leurs tournettes individuelles, les vases doivent être déplacés pour se succéder sur l'unique tour. De plus, la grande hauteur des vases oblige le potier à se tenir debout pour façonner les derniers étages des pithoi, ce qui est impossible sur un tour électrique. De ce fait, les grandes jarres sont terminées sur les anciennes tournettes actionnées à la main par un aide (fig. 5). La procédure de façonnage des pithoi est donc restée inchangée, à quelques opérations 
près. C'est surtout dans le montage des pièces de taille moyenne que le tour électrique présente des avantages.

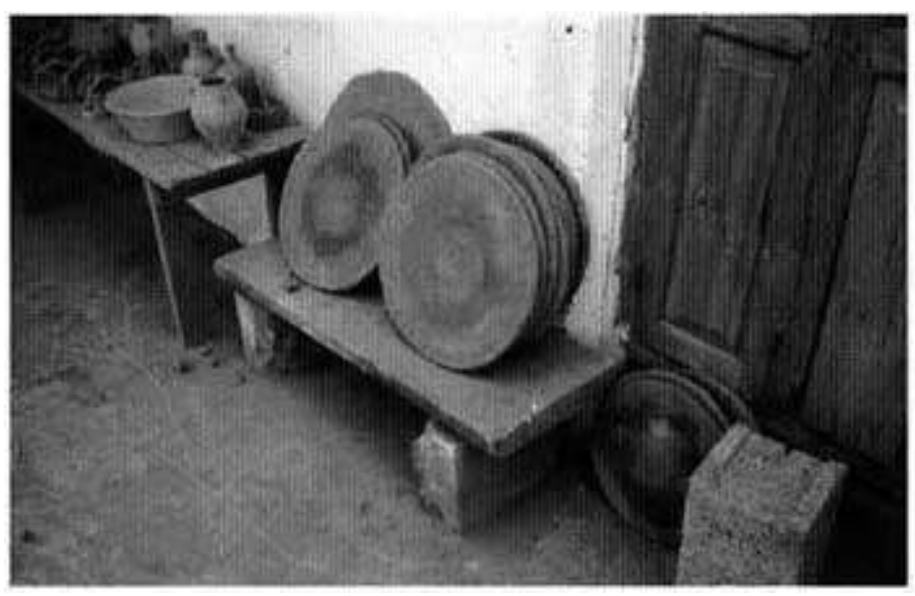

Photo 2. Atelier de Nikos K. Rondeaux en schiste

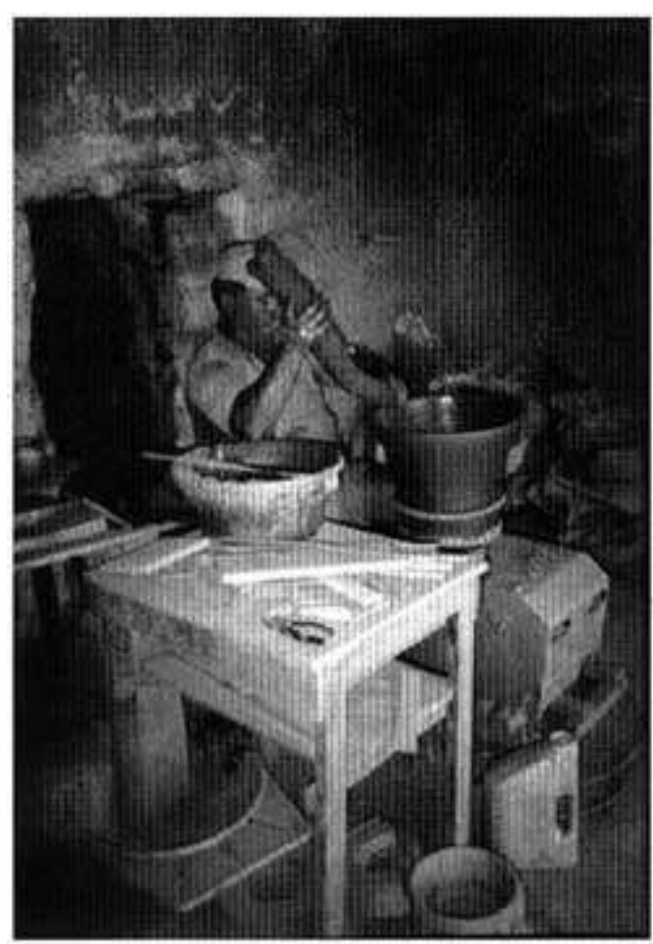


Photo 3. Atelier de Nikos K. Façonnage des grands vases : mise en place du colombin

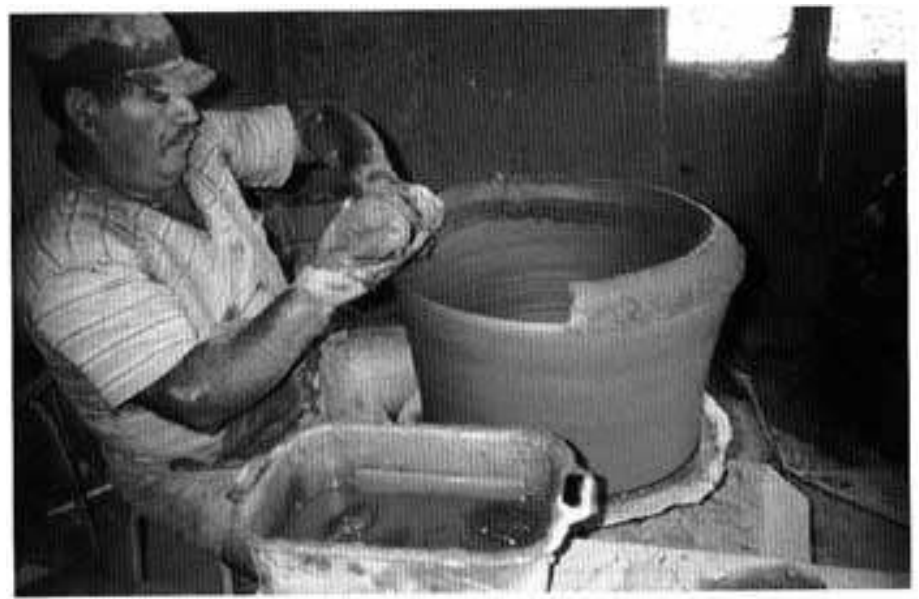

Photo 4. Atelier de Nikos K. Façonnage des grands vases : amincissement du colombin et montage d'un étage de la paroi

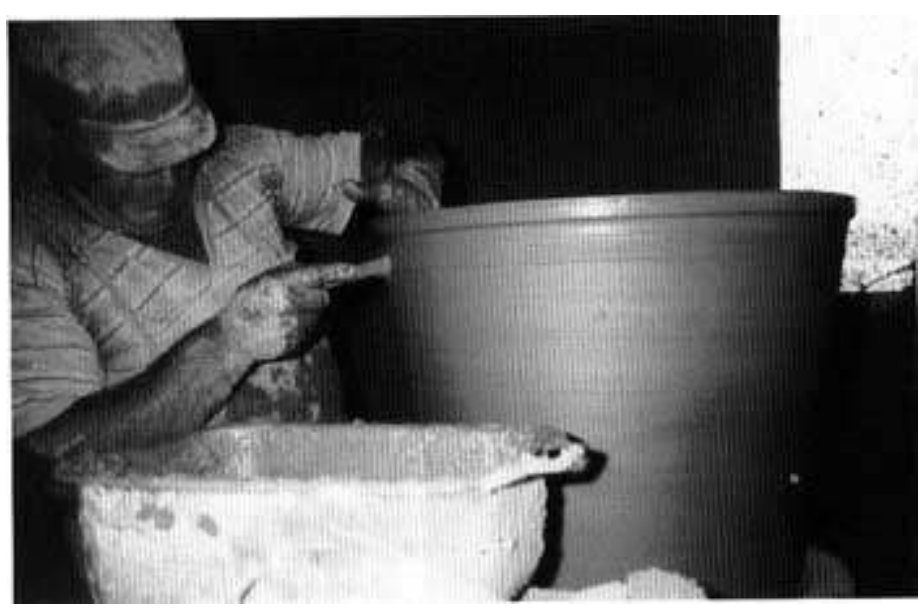


Photo 5. Atelier de Nikos K. Façonnage des grands vases : mise en forme d'un étage de la paroi

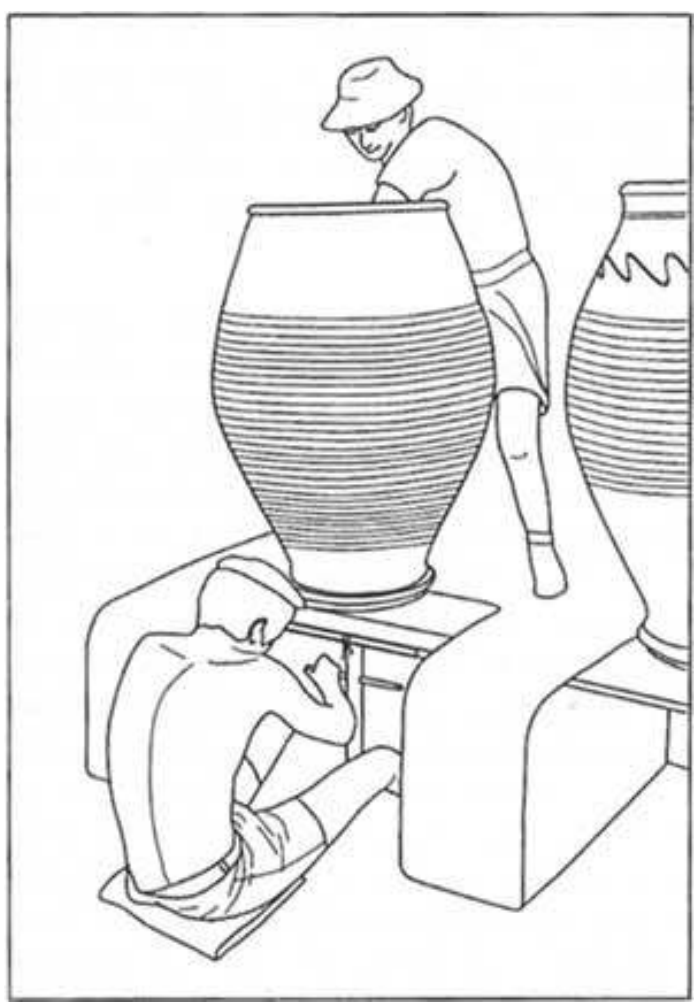

Figure 5. Finition d'une grande jarre sur une tournette manuelle (dessin Ch Vogt, d'après Vallianos \& Padouva $1986: 83$ )

Façonnage des éléments de la panse

Monté par étages successifs comme on le fait pour les murs en pisé, chaque nouvel élément de la paroi du vase repose sur le précédent (fig. 6). Celui-ci doit donc être suffisamment sec et dur pour pouvoir supporter son poids, soit 7 ou 8 kilos. Le séchage de la paroi -qui dépend naturellement de la chaleur et de l'hygrométrie de l'air-peut prendre deux heures en été et le double ou le triple en hiver. Toutefois, en été au moins, ce délai est annulé par le montage en série car Nikos K., évidemment, façonne plusieurs jarres simultanément. Il passe de l'une à l'autre en montant à chaque fois un nouvel élément du vase : d'abord toutes les assises, puis les bases, puis les premiers étages, les seconds, etc. (fig. 7). Ainsi, par beau temps, le premier pithos est suffisamment sec pour supporter un nouvel étage lorsque le potier a fait le tour des six ou huit autres vases. De plus, pour éviter les fissures et les affaissements, la panse des vases est parfois cerclée de plusieurs tours de corde ${ }^{12}$. Enfin, le joint entre deux éléments de la paroi est renforcé par un bourrelet de pâte creusé de cannelures faites du bout des doigts. Étage après étage, la paroi du pithos s'élève ainsi par superposition d'éléments identiques (Psaropoulou 1984: 18-19). Les plus grands pithoi montés par Nikos K. se composent de neuf étages successifs pour une hauteur d'un mètre et demi.

Façonnage du col et des anses, décoration

Le col du vase est obtenu par simple étirement du bord du dernier étage. La lèvre n'est en fait qu'un épaississement modelé, puis régularisé avec l'estèque. Elle est toujours simple, parfois saillante ou rentrante. Sa terminaison horizontale permet la pose d'un couvercle en céramique ou en bois. Les jarres sont presque toujours décorées de motifs 
incisés ou imprimés dans la pâte fraîche, le plus souvent des lignes horizontales et ondulées ou des bandes peignées ondulées. On trouve aussi des incisions en arêtes de poisson destinées à masquer les raccords des éléments de la paroi. Pour les réaliser, le potier utilise simplement le côté coupant de l'estèque. Les bandes peignées étaient autrefois obtenues à l'aide d'un morceau de bois dentelé mais aujourd'hui, cet outil est remplacé par un tronçon de gros peigne à cheveux. Les motifs imprimés sont obtenus par application de sceaux ou cachets en bois sculptés de motifs géométriques, symboliques, naturalistes et encore souvent, de croix et de citations religieuses. Les anses sont ajoutées en dernier, sur le vase déjà décoré. Elles sont faites d'un tronçon de colombin dont chaque extrémité est collée avec un peu de barbotine puis fixée sur la paroi par une simple pression des doigts. Il faut noter que le nombre d'anses augmente en fonction de la hauteur du pithos : plus il est grand, plus il y en a (photo 6).

Séchage des vases

Avant d'être enfournés pour la cuisson, les pithoi doivent être parfaitement secs. Leur séchage, lent pour éviter les fissures, a toujours lieu à l'ombre. En saison humide par contre, le potier doit parfois accélérer le séchage en brûlant des touffes de brindilles à l'intérieur même des vases (Hampe \& Winter 1962, frontispice). Chez Nilcos K., la construction d'un hangar spécial a maintenant résolu ce problème.

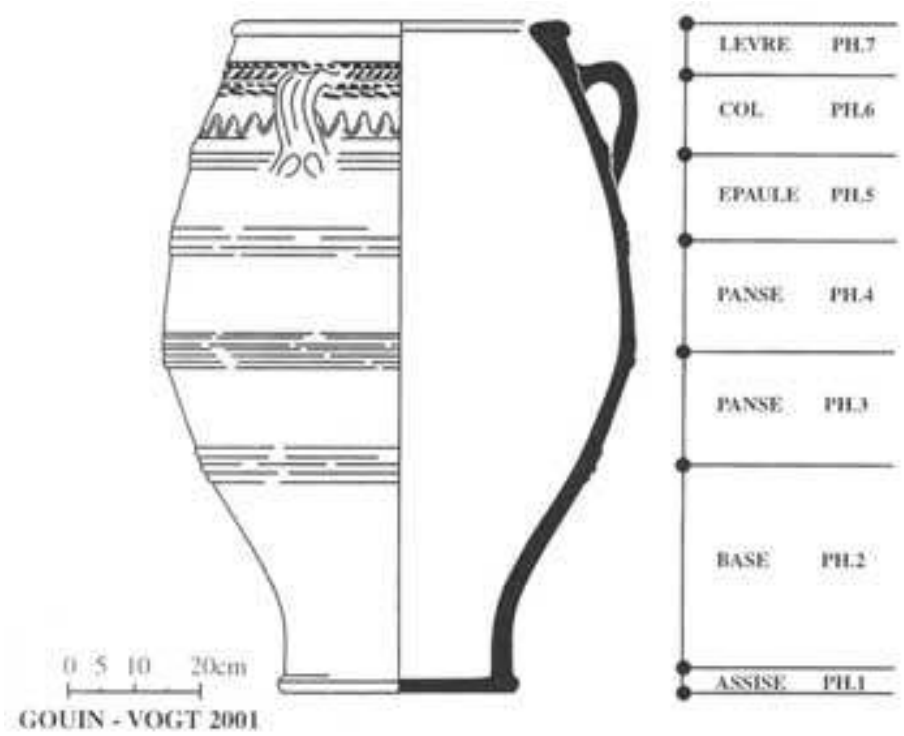


Figure 6. Principales phases du montage d'une grande jarre (dessin Ph. Gouin et Ch. Vogt)

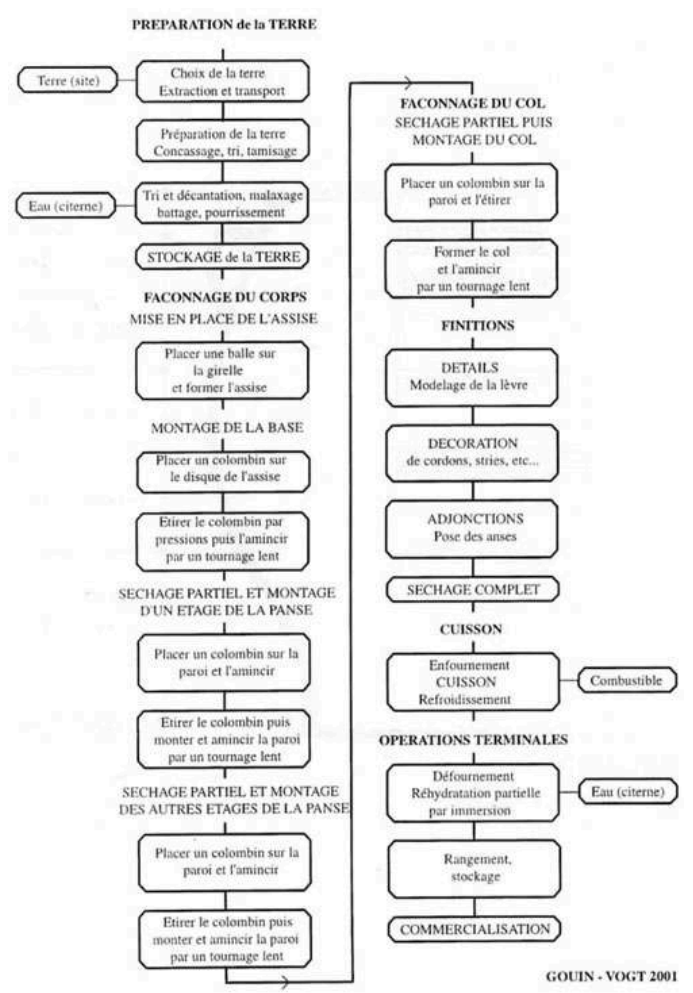

Figure 7. Diagramme de fabrication des grandes jarres (dessin Ph. Gouin et Ch. Vogt)

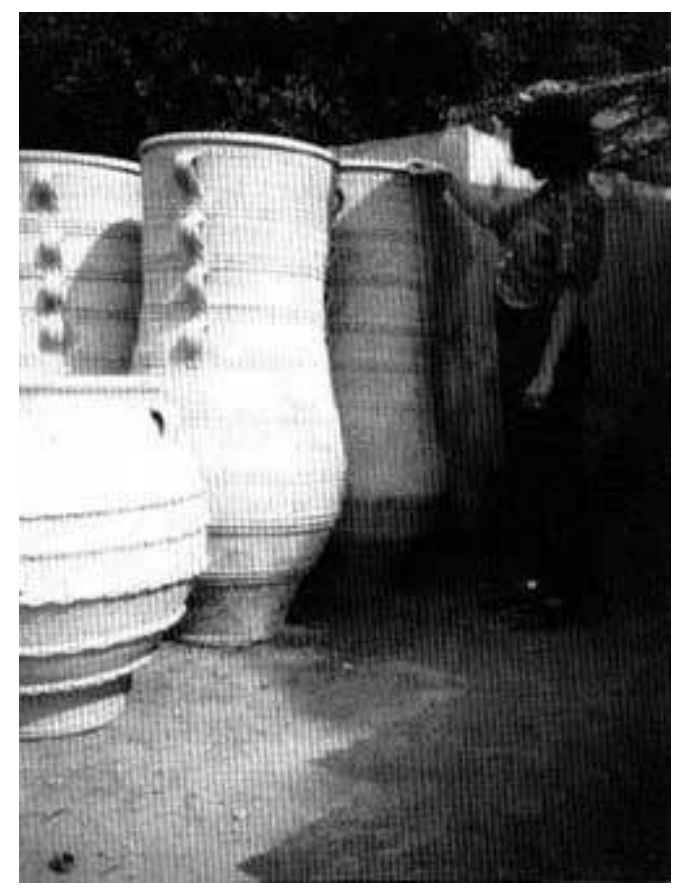

Photo 6. Atelier de Nikos K. Grande jarre à 12 anses

Cuisson des vasesOrigines des combustibles

21 La Crète n'a pas toujours été aride. La présence d'une cité aussi importante qu'Eleutherna prouve que la région était dans l'Antiquité assez humide et qu'elle 
pouvait nourrir une population considérable ${ }^{13}$. De même, tous les voyageurs qui passèrent par la Crète à la fin du Moyen Âge décrivent l'île comme verdoyante et boisée (Warren 1972). C'est seulement depuis quelques siècles que les montagnes, déboisées par les conquérants étrangers puis par les bergers crétois, sont devenues arides et le bois rare (Vogt 2000). En effet, une fois dégradé, le tapis végétal se reconstitue difficilement car l'île se trouve, ne l'oublions pas, à la même latitude que la Tunisie et jouit donc en été d'un climat brûlant et sec. Seuls les plaines côtières septentrionales et les plateaux, comme la Messara et le Lasithi, permettent aujourd'hui une agriculture d'une certaine importance. Les parties du relief non plantées d'oliviers ou de vignes restent incultes et sont livrées aux moutons et aux chèvres.

Récemment encore, trouver du combustible n'était donc pas le moindre problème du potier crétois qui ne disposait pas, comme aujourd'hui, de sciures et de copeaux industriels. La cuisson d'une fournée de dix ou douze jarres consommait en effet une cinquantaine de fagots de branchages divers, de 50 kilos chacun. La part de responsabilité des potiers dans le déboisement d'une région était donc considérable, même s'ils brûlaient aussi tout ce qui pouvait l'être comme la paille, les broussailles et même, en saison, les tourteaux de noyaux d'olives pressées.

\section{Fonctionnement des fours}

Nikos K. cuit aujourd'hui ses productions dans un four rectangulaire moderne construit en briques réfractaires et chauffé avec de la sciure de bois industrielle et divers déchets. Mais certaines fournées spéciales sont encore cuites de façon traditionnelle dans l'un des antiques fours ronds. Parfaitement conservés, ces fours bordent encore la route, aujourd'hui goudronnée, qui traverse l'ancien village de potiers (voir fig. 3). Malheureusement, cette route va être élargie pour faciliter le passage des autocars de touristes et les fours seront sans doute détruits un jour, malgré les protestations des villageois attachés à leur passé. Tous ces anciens fours sont du même type (fig. 8). Leur épaisse paroi circulaire en maçonnerie de moellons entoure le foyer surmonté d'une chambre de cuisson un peu plus large. Des tuyères percées dans la sole voûtée font communiquer les deux parties. Le four ne possède ni coupole, ni porte fixe. Durant la cuisson, la fournée est seulement recouverte de larges tessons de jarres et d'une couche de terre. Une cheminée, ménagée au centre de cette couverture, permet aux fumées de s'échapper. La chambre de cuisson d'un de ces fours ne peut contenir plus d'une douzaine de pithoi rangés verticalement sur deux couches, l'assise de l'un étant posée sur l'ouverture de l'autre. Les interstices sont généralement remplis de vases plus petits (photo 7). La cuisson traditionnelle dure environ huit heures pendant lesquelles le foyer doit être sans cesse alimenté. La température intérieure du four atteint $1000^{\circ} \mathrm{C}$, température normale pour des argiles communes. La cuisson terminée, on laisse l'ensemble se refroidir suffisamment pour pouvoir dégager les vases cuits. Du chargement au déchargement du four, l'opération prend deux ou trois jours en été et un ou deux en hiver. Ce délai est difficilement compressible car un refroidissement trop rapide risquerait de fendre certains vases. Dans les meilleurs cas, cinq à dix pour cent des vases sont cassés ou ratés durant leur cuisson. Pourtant, tous ne sont pas perdus car, même fendus ou fondus, ils peuvent encore être vendus hors de Grèce. En effet, les vases défectueux sont particulièrement recherchés par les clients étrangers qui y voient une preuve d'authenticité. Bien qu'il y trouve bénéfice, cela afflige notre maitre potier qui est resté attaché à la belle ouvrage. 


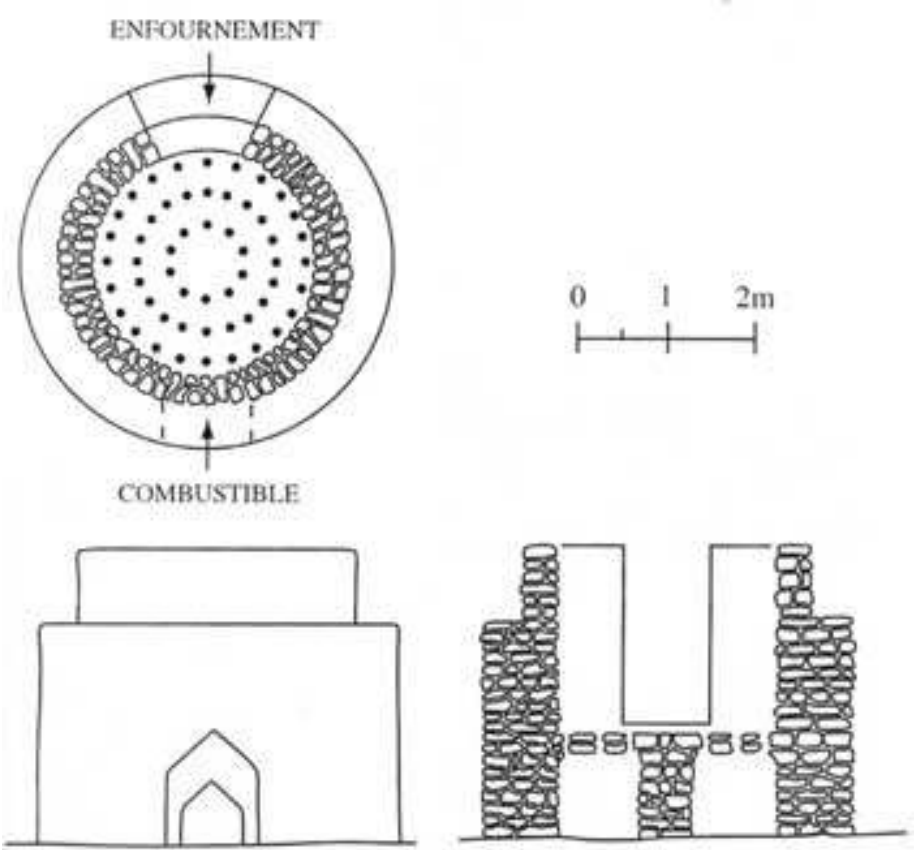

Figure 8 : Fonctionnement des fours

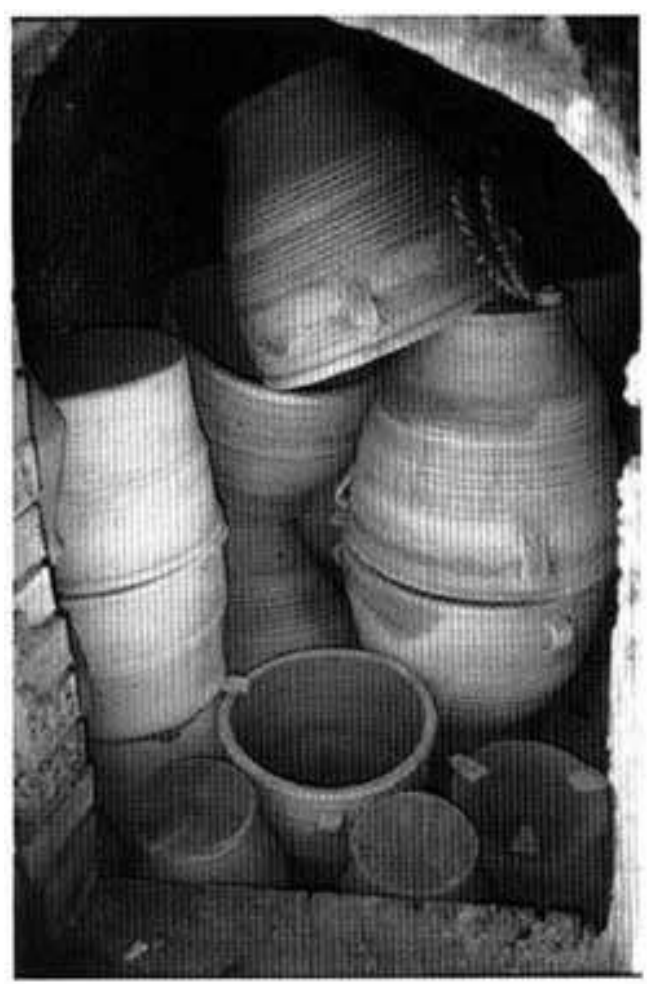

Photo 7. Atelier de Nikos K. Chargement du four

« Réhydrataion » et stockage des produits

24 Après cuisson et complet refroidissement, Nikos K. laisse les vases tremper quelques jours dans des grandes cuves remplies d'eau additionnée de chaux. Il nous a assuré que ce trempage les rendait moins poreux ${ }^{14}$. La place exigée par le séchage, le stockage des 
vases cuits et le trempage est considérable puisqu'elle prend $80 \%$ de l'hectare de terrain plat occupé par les installations. De plus, une large allée centrale doit rester libre pour permettre la circulation des véhicules qui apportent la terre et chargent les vases. Mais ici le stockage ne pose que des problèmes de manutention et non de place, puisque les installations sont implantées en plein maquis, au-delà même des vieux ateliers abandonnés. Elles pourraient donc s'agrandir si nécessaire.

\section{La diffusion}

Bien que chaque partie de l'île ait autrefois possédé ses propres ateliers, les jarres de Margaritès étaient vendues et utilisées dans toute la Crète (Psaropoulou 1996). Jusqu'à la Seconde Guerre mondiale, ces vases faisaient aussi l'objet d'un important commerce à longue distance en Méditerranée orientale. On les retrouvait non seulement dans les îles de l'Égée qui ne possédaient pas d'ateliers ou de terre à pots (Blitzer 1990 : carte 703) mais aussi jusque dans les Balkans, en Turquie et au Levant où les ateliers produisant de très grands récipients étaient rares (Güner 1988). Aujourd'hui, le réseau de clientèle a bien changé. Il s'est orienté vers l'Europe et particulièrement vers l'Allemagne où près de $70 \%$ des produits sont vendus directement comme objets de décoration. Le reste de la production fournit les boutiques de curiosités d'Athènes et le marché local du mobilier urbain ${ }^{15}$ (photo 8 ).

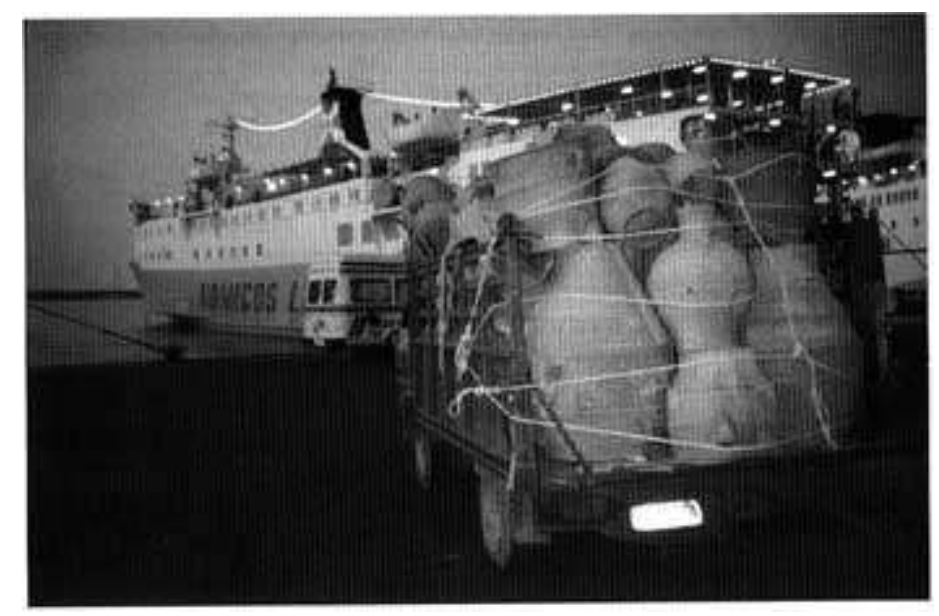

Photo 8. Héraklion. Embarquement des vases pour le continent

Déjà détournées de leur usage alimentaire, les vénérables jarres des grand-mères crétoises où s'entassaient jadis toutes les richesses familiales ont donc été bien près de disparaître avec leur technologie plusieurs fois millénaire ${ }^{16}$.

Nous remercions très chaleureusement le Professeur P. Thémélis (Université de Crète) ainsi que Mme X Hiloudaki (Musée archéologique de Réthymno) et M. N. Lianéris (Archéologue, Athènes, Réthymno), qui ont traduit nos entretiens avec le maître-potier N Kavgalakis. Nos remerciements vont aussi à M. H. Vallianos, Directeur du Musée Ethnographique de Vori, dont les travaux (Vallianos et Padouva 1986), avec ceux de R. Hampe et A. Winter (1962), ont servi de base à notre étude. Notre gratitude va enfin à Mme. R. Stadaki, Fondatrice et Conservatrice du Musée ethnographique de Réthymno, qui nous a largement ouvert ses collections de céramiques traditionnelles.

Clichés : Ph. Gouin et Ch. Vogt. 


\section{BIBLIOGRAPHIE}

Aloupi, Éléni, Kilikoglou Vassilis \& Peter M. Day

2000. « Provenance and technological characterisation of fine tableware», pp. 229-235, in P.

Thémélis (ed.), Protobyzantini Eleutherna. Tome 1 B. Réthymno : Panepistimio Kritis.

Blitzer, Harriet

1990. « Koroneika storage-jar production and trade in the traditional

Aegean », Hesperia 59 : 675-711 et pl. 99-112.

Brissaud, Philippe

1982. Les ateliers de potiers de la région de Louqsor. Le Caire : Institut Français d'Archéologie

Orientale.

Centlivres-Demont, Micheline

1971. Une communauté de potiers en Iran. Wiesbaden : L. Reichert.

Gelbert, Agnès

1997. « Tour et tournette en Espagne : recherche de macrotraces significatives des différentes techniques et méthodes de façonnage ", pp. 59-91, in Terre cuite et société. La céramique, document technique, économique, culturel (Actes des XIVe Rencontres internationales d'Archéologie et d'Histoire d'Antibes). Juan-les-Pins : APDCA.

Gouin, Philippe

1996. «O Skylos kai o Kynikos. Me Aformi to Pithari ton Diogeni », Ariandi 8 : 101-108.

1997. « Ancient oriental dairy techniques derived from archaeological evidence », Food and Foodways 7.3 : 157-188.

Gouin, Philippe \& Christine Vogt

1996. « Origine, Apogée et Déclin des potiers de Margaritès (Crète centrale) », pp. 55-68, in I. Gavrilaki (ed.), Kerameika ergastiria stin Kriti apo tin arkhaiotita os simera, (Actes du colloque de Margaritès 1995). Réthymno : Panepistimio Kritis.

2000. «Quarrymen and Potters », pp. 210-205, in P. Thémélis (ed.), Protobyzantini Eletherna Tome 1 B : 201-205. Réthymno : Panepistimio Kritis.

Güner, Güngör

1988. Anadolu'da yasamakta olan ilkel çömlekçlik. Istanbul : Marmara Universitesi.

Hampe, Roland

1963. « Die Pithostöpfer von Kreta », Kritikn klnonika IE-IST 3 (1962) : 213-227.

Hampe, Roland \& Adam Winter

1962. Bel Töpfern und Töpferinnen in Kreta Messenien und Zypern. Mayence : von Zabern.

Kalpaxis, Thanasos, Purtwàngler, Andréas \& Alain Schnapp

1994. Eleuthernall, 2. Réthymno : Ekdoscis Panepistimiou Kritis.

Jones, R. E.

1986. « Traditional potters of contemporary Grcece and Cyprus », pp. 849880, in R.E. Jones (ed.), Greek and Cyprint Pottery. Fitch Laboratory Occasional Papers 1. Athènes : British School of Athens.

Leroi-Gourhan, André

1943. Milieu et technique (édition 1971). Paris : Albin Michel. 
Psaropoulou, Betti

1984. Last potters of the East Aegean. Athènes : Livre à compte d'Auteur.

1996. «Ta keramika ergastiria ton teleutaion 10 xomon stin Kriti », pp. 101-138, in Kerameika ergastiria stin Kriti apo tin arkhaiotita os simera (Actes du ler Colloque international 1995 de Margaritès, Crète). Réthymno : Istoriki Laografiki Etaireia Rethymnis.

Roux, Valentine

1994. « La technique du tournage : définition et reconnaissance par les macrotraces », pp. 45-58, in Terre cuite et société. La céramique, document technique, économique et culturel (XlVèmes Rencontres internationales d'Archéologie et d'Histoire d'Antibes). Juan les Pins : APDCA.

Rye, Owen S. \& Clifford Evans

1976. Traditional Pottery techniques of Pakistan. Washington : Smithsonian Institution Press.

Thémélis, Pétras (ed.)

2000. Protobyzantini Eleutherna. Tome I B. Réthymno : Panepistimio Kritis.

Vallianos, Khristophoros et Maria Padouva

1986. Ta Kritika Aggeia ton I9ou Kai 20ou Azona. Athènes : Mouzeio Kritikis Ethnologias.

Vogt, Christine

2000.« The Early Byzantine Pottery », pp. 13-200, in P. Thémélis (ed.), Protobyzantini Eleutherna. Tome 1B. Réthymno : Panepistimio Kritis.

Voyatzoglou, Maria

1974. « The jar Makers of Thrapsano in Crete », Expedition $16.1: 18-14$.

Warren, Peter

1972. « 16th, 17th and 18th century british travellers in Crete », kritika Khronica : 65-92. Iraklcion : Examiniaia Episrimoniki Ekdosis.

\section{NOTES}

1. L'étude des potiers de Margaritès a été réalisée en 1992-94, au cours de trois missions ethno-archéologiques financées conjointement par la Fondation Nationale de la Recherche Hellénique, le Centre National de la Recherche Scientifique et l'École française d'Athènes.

2. Le témoignage technique est précieux car c'est sur lui que repose la possibilité de ne pas confondre ce que nous supposons être les premiers pas de l'humanité avec ce que nous en savons objectivement (Leroi-Gourhan 1943 : 10).

3. On se souvient que Diogène y vécut le temps d'une démonstration philisophique (Gouin 1996).

4. Les pithoi sont lavés à l'eau bouillante mêlée de cendres de bois (effet détergent) et frottés avec un tampon de branchettes de thym (effet mécanique et antiseptique).

5. On remarquera cependant que la couleur rouge-brique, c'est-à-dire terre cuite, reste la plus appréciée.

6. En effet, la disparition du stockage en jarres s'accompagne d'un changement partiel des modes de production et de consommation alimentaires.

7. Le nom d'Eleutherna figure dans les textes antiques. Les fouilles (les archéologues grecs de l'Université de Crète ont aussi montré que le site était occupé dès le Minoen. 
8. La visite du site est d'une grande tristesse car on ne peut éviter de penser au drame des artisans, toujours pauvres et souvent âgés qui durent abandonner leur outil de travail ancestral. Les céramistes d'art qui les ont remplacés se sont tous établis dans des maisons restaurées du village, à proximité des touristes. Leur production est uniquement décorative.

9. L'exploitation des carrières, réservée aux seuls potiers, est réglementée par une assemblée d'artisans et de notables du village.

10. La charge d'un âne ne dépassait pas 80 kilos de terre à chaque voyage.

11. Il s'agit approximativentent du rouge-clair N 39 de la charte Cailleux qui correspond au 2,5 YR 6/8 de la charte Munsell.

12. La marque de cette corde se retrouve fréquemment sur la panse des grands vases culinaires antiques.

13. Les vases citernes creusés sous l'acropole d'Eleutherna, qui alimentaient thermes et fontaines, pouvaient contenir plusieurs milliers de mètres cube d'eau...

14. Le calcaire calcinéparlacuissonformepeutêtre avec l'argile une sortede « chaux hydraulique "quidurcitlorsdelaréhydradation.

15. Ces fortes demandes étrangères et locales ont sans doute sauvé les pitboi. En effet, leurs prix ont de ce fait beaucoup augmenté et leur fabrication est redevenue rentable. Chez le potier, les jarres à 4 anses de 200 litres valentunecentained'eurosetcellesde 500 litresà douzeanses,àpeuprèsledouble. Elles sont vendues jusqu'à 3 fois plus cher par les antiquaires d'Athènes.

16. Cette étude achevée, nous nous sommes aperçus que toutes nos observations, à quelques détails près, auraient pu être obtenues par analyse déductive des structures et du matériel d'une fouille archéologique soigneuse. Cela tendrait à prouver que l'archéologue passe souvent à côté d'informations « ethnographiques " parce que, faute de modèles, il ne lui vient pas à l'idée d'en exploiter les données.

\section{RÉSUMÉS}

À l'écart des touristes, quelques potiers crétois fabriquent encore comme autrefois une poterie rustique destinée aux usages domestiques. C'est le cas à Margaritès, en Crète centrale, où il est possible d'observer le façonnage des grands vases de stockage, les pithoi, déjà en usage quinze siècles plus tôt dans la cité antique voisine d'Eleutherna. L'étude conjointe et comparative des deux productions céramiques, l'ancienne et la traditionnelle, nous a ainsi permis de reconstituer les techniques de fabrication des potiers d'autrefois. Elle nous apporte aussi de nombreuses données sur l'économie des villages crétois durant la période, encore peu connue, comprise entre la fin de l'Antiquité et l'occupation vénitienne (VIIe-XIIIe siècles).

The pithoi of Margaritès (Crete). Technical data and historical overview

Away from the tourists, Cretan potters still produce traditional crockery for domestic use. It is the case in Margaritès (central Crete) where one can observe the making of huge jars for food storage, called pithoi. These were already in use fifteen centuries ago in the neighbouring antique city of Eleutherna. The joint and comparative study of antique and traditional ceramic productions enabled to reconstitute the techniques fo the ancient potters. Such approach also 
brought important data on the economy of Cretan villages of this poorly documented historical period spanning from the end of Antiquity and the Venitian occupation (7th-13th centuries).

Los pithoi de Margarites (Creta). Datos técnicos y compendio histórico

Fuera de los circuitos turísticos, unos cuantos alfareros cretenses siguen confeccionando objetos rústicos dedicados a usos domésticos, tal y como se hacía en el pasado. En Margarites (Creta central), es posible observar la fabricación de grandes vasijas destinadas a conservar alimentos, los pithoi, ya empleadas hace quince siglos en Eleutherna, una ciudad antigua próxima a este pueblo. El estudio simultáneo y comparativo de las dos producciones cerámicas -la antigua y la tradicional- ha permitido reconstituir las técnicas de fabricación de los alfareros de la Antigüedad. También nos proporciona numerosos datos acerca de la economía de los pueblos cretenses durante la época - aún poco conocida- comprendida entre el fin de la Antigüedad y la ocupación veneciana.

\section{INDEX}

Mots-clés : chaîne opératoire, Antiquité, céramiques, Crète, ethno-archéologie, Grèce, potiers

Keywords : operational sequences, Antiquity, ceramic, Crete, ethno-archaeology, Greece, potters

\section{AUTEURS}

\section{PHILIPPE GOUIN}

Centre d'Archéologie Orientale de l'Université de Paris I (Panthéon-Sorbonne), 3 rue Michelet, Paris et CNRS, UMR 7041, Maison de l'Archéologie et de l'Ethnologie, ll allée de l'Université, 92023 Nanterre Cedex.

\section{CHRISTINE VOGT}

Centre d'Archéologie Orientale de l'Université de Paris I (Panthéon-Sorbonne), 3 rue Michelet, Paris et CNRS, UMR 7041, Maison de l'Archéologie et de l'Ethnologie, ll allée de l'Université, 92023 Nanterre Cedex. 